She came as an out-patient under Dr. Squarey in August, 1870, with a tumour in right iliac fossa, extending an inch beyond the umbilicus; it seems elastic and rather tender; indistinct fluctuation and dulness on percussion; above the pubes, on the left side, a softer and more elastic mass is felt, not distinctly connected with that before mentioned. On vaginal examination a tumour is felt in front, and on each side of the uterus, but giving the impression of not being connected with the tumour felt in abdomen.

On admission the girth at the umbilicus was nearly 32 inches; the tumour reached $1 \frac{1}{2}$ inch above umbilicus, freely movable. Uterus high up in pelvis; no tumour felt per vaginam ; urine normal.

Operation, March 18th.-The tumour, which sprang from left ovary, was found free from adhesions; four pints of viscid, slightly milky fluid were removed through the trocar; the pedicle was tied as in other cases, and the tumour removed. A closely compacted cystic tumour, about the size of a large pear, found in connexion with each ovary, was removed, and some little cysts in the left broad ligament were opened, and the contents evacuated. The wound was closed, and the abdomen covered with linseed and laudanum poultices, which were changed every four hours.

The patient recovered without a bad symptom. A trace of albumen appeared on the third day. Sanguineous discharge came on the fourth day, and lasted in small quantities four days. The sutures were removed on the sixth day. The wound was perfectly united, but there was some little suppuration afterwards from one of the upper sutures.

A fortnight after the operation the uterus was found central, fixed by deposit around, but especially in the posterior half of the pelvis, quite elastic, and somewhat bulg. ing. Six days afterwards the lump was only felt as a hard ridge behind the uterus.

The patient was discharged thirty-two days after operation. She came as out-patient August 1st, when she stated that she continued to menstruate quite regularly, and has sexual desire as much as ever.

CASE 3. Multilocular cyst; history of three months; recovery. C. T-, aged thirty, single, admitted under Mr. Heath March 22nd, 1871. The catamenia began at seventeen years of age; very profuse; lasting a week; the last interval being only a fortnight; no pain. Eight weeks ago her attention was attracted to the abdomen by feeling pain in the left side, when she found it very hard and resistant and larger than usual. Dr. Robson, of Iver, who has attended her, said that it had increased two inches in three weeks. She thinks she has noticed her abdomen large for three months. Appetite good. Nearly the whole of ber abdomen is occupied by a tumour rather more to the left than the right side; dull on percussion; felt rising out of the pelvis on the right side; moderately smooth on the surface; harder and nodulated in the left hypochondrium ; the whole right side of the tumour is less hard and more elastic than the right. No distinct fluctuation; no enlargement of superficial veins. Girth at umbilicus, 32 in.

Vaginal examination. - The cervix felt of normal length, rather soft; directed downwards, backwards, and to the left. Sound three and a half inches upwards and slightly to the right, quite in front of the tumour, which is felt posteriorly and very close to the uterus. 'The tumour in the pelvis is the same as that in the abdomen. Urine normal.

A consultation was held, and the almost universal opinion was that the case was more likely to be a uterine than an ovarian tumour; but an exploratory incision was recommended, as patient was getting unfit to do any work.

Operation, March 25th.-The tumour was found to be an extensively multilocular cyst of the right ovary, with a very broad pedicle and no adhesions. The uterus felt healthy, not particularly enlarged. The fluid removed, which was small in quantity, was very viscid, and dark-brown in colour. The cyst, which was very solid, weighed $7 \mathrm{lb}$.

The urine for the first twenty-four bours contained a trace of albumen. The sutures were removed on the fifth day. The wound was quite united, but gaped a little five days afterwards, and did not perfectly heal for another month, when she was discharged (May 11th).

Aug. 23rd.-Well and strong; cicatrix sound; pain in right iliac region after exertion.

We must reserve the continuation of this report until our next number.

\section{SAMARITAN HOSPITAL.}

ULCER OF THE STOMACH ; RUPTURE OF THE HEART. (Under the care of Dr. WiLtshiRe.)

C. B-, a widow, aged fifty-seven, came to the hospital on Jan. 16th, 1872, complaining of a "bilious attack." She has pain between the shoulders, distress and tenderness at the epigastrium, and flatus and vomiting after food, which always excites pain ; has occasionally vomited "black stuff," and has melæna ; bowels open; urine high-coloured, but not thick; pulse very feeble, A daughter who accompanied the patient said that a few nights before her mother had had a curious attack, which, from the description given, might have been of an epileptic character.

An vlcer of the stomach was diagnosed, and a mixture of bismuth, soda, hydrocyanic acid, and calumba was prescribed.

Three days afterwards the patient sent to say that she was better, but the day being wet she had not ventured out. The night following the patient felt so much better that she partook of meat for supper, and afterwards ate some figs. An hour or two after this, when about to undress for bed, she was suddenly seized with "faintness" and expired.

At the post-mortem examination (at which, owing to the courtesy of Mr. Dowd, the gentleman who was called to the patient when she died, Dr. Wiltshire was enabled to be present) the pericardium was found to be distended with blood, which had escaped from a rupture, three-quarters of an inch long, in the right ventricle. The muscular tissue of the heart was in a state of marked fatty degeneration. The valves, though the seat of some atheromatous change, were competent. In the posterior wall of the stomach, about the beginning of the pyloric third, were found three small ulcers, their surfaces being covered with discoloured blood. Each was about the size of a split-pea, and they were grouped around the cicatrix of an old ulcer, which measured three-quarters of an inch by half an inch. The other organs of the body presented nothing notable.

The sudden death of the patient from ruptured heart permitted a verification of the diagnosis of gastric ulcer made during life; and the case affords another instance of the healing of large ulcers and subsequent invasion of the organ with a like lesion.



\section{PATHOLOGICAL SOCIETY OF LONDON.}

Tumsdax, February 20Th, 1872.

Mr. John Hurton, F.R.S., President, in the Chatr.

The President intimated to the Society that the Council had recommended that portions of certain evenings should be set aside for the discussion of abstract questions. Any member of the Society might make a prearrangement with the secretaries for the exhibition of specimens or drawings illustrative of some particular point, and, due notice having been given beforehand by means of the usual channels, there could be little doubt that very valuable discussions would result from the observance of this plan. The time had certainly arrived when the Society might make use of the enormous amount of valuable material accumulated in the volumes of its Transactions, and attempt to deduce therefrom important generalisations.

A report by the Morbid Growths Committee on $\mathbf{M r}$. Hutchinson's case of tumours growing between the tibia and fibula stated that the tumours in question belonged to the round-celled division of Virchow's class of sarcomata

Dr. C. T. WILliams exhibited a patient whose chest showed an unusual amount of depression in the lower sternal region. The deformity was congenital, and appeared like an exag. geration of the deformity sometimes met with in cobblers. Besides the sternal depression, there was some general flat. tening of the chest, and the shoulders were thrown considerably forwards; but there was no true curvature of the spine. The heart was displaced laterally, and the liver drawn upwards. Dr. Williams's account of this patient was accom. 